\title{
FRACTALS FOR COMPLEXITY ANALYSIS OF DIABETIC RETINOPATHY IN RETINAL VASCULATURE IMAGES
}

\author{
Nazneen Akhter ${ }^{1}$, Yogesh Rajput ${ }^{2}$, Sumegh Tharewal $^{3}$, K. V. Kale ${ }^{4}$, Ramesh Manza \\ ${ }^{1,2,3}$ Research Fellow, ${ }^{4}$ Professor \& Head, ${ }^{5}$ Asst. Professor, Dept. of CS \& IT, Dr. B. A. M. University, Maharashtra, India
}

\begin{abstract}
Arterial pattern and morphology of distribution is damaged because of diabetes resulting in retinal vasculature deformation. This aspect is studied in terms of quantification of the degree of complexity associated with the distribution of blood vessels in eye for healthy and diabetic humans. Retina images of fifteen healthy subjects are compared with those of diabetic subjects. It is found that the increased complexity of structure and texture of the diabetic subjects results in a higher fractal dimension as compared to those of healthy subjects. Also the blood vessel patterns, both for healthy and diabetic subjects show self-similarity and scale invariance and hence the patterns are fractals. For the purpose of characterization of the irregular patterns of blood vessels in retina, box counting technique is used for the estimation of fractal dimension. A GUI based program is developed in Matlab for implementation of box counting technique and determination of fractal dimensions. Fractal dimension of retina images for diabetic subjects show higher fractal dimensions indicating higher degree of structural complexity associated with the image whereas images of healthy subjects show a lower value of fractal dimension indicating limited complexity of structure. It is shown that fractal dimension can be used to distinguish diabetic subjects from healthy subjects and hence this technique could be used in diagnosis of diabetes using images of retina. It is interesting that during other diagnostic procedures related to retina images, this information can be generated as additional information adding value to the diagnostic procedures. Details of implementation of the technique are presented.
\end{abstract}

Keywords: Diabetic Retinopathy, Fractal Dimension, Box Counting, Segmentation, Image Processing

\section{INTRODUCTION}

Diabetic retinopathy is damage to the retina caused by complications of diabetes, which can eventually lead to blindness. which affects up to 80 percent of all patients who have had diabetes for 10 years or more. ${ }^{[1]}$ Despite these intimidating statistics, research indicates that at least $90 \%$ of these new cases could be reduced if there was proper and vigilant treatment and monitoring of the eyes. ${ }^{[2]}$ The longer a person has diabetes, the higher his or her chances of developing diabetic retinopathy. ${ }^{[3]}$ Diabetic retinopathy is one of the foremost frequent causes of blindness world-wide. In India, it was the 17th cause of blindness 20 years ago but has now ascended to the 6th position. Diabetic retinopathy has been identified as one of the significant causes of blindness or vision impairment in India. Though cataract is still the leading cause of blindness, the intense work under the National Programme for Control of Blindness (NPCB) with the support of international non-governmental organisations has brought down its contribution to blindness. There are 41 million of diabetics in India at present and every diabetic is a potential candidate for loss of vision due to diabetic retinopathy. This number is poised to increase significantly. Thus it is an appropriate time now to concentrate on diabetic retinopathy and bring the problem under control ${ }^{[4]}$. However, we have to recognize that the issues in managing vision impairment due to diabetic retinopathy are different from cataract. While cataract blindness is curable by a simple one time surgical intervention, diabetic retinopathy encompasses a multitude of problems and can be prevented if detected early and treated. In the first stage which is called non-proliferative diabetic retinopathy (NPDR) there are no symptoms. The only way to detect NPDR is by fundus photography (Retinal fundus images, using a digital fundus camera).

The retina is the only location where blood vessels can be directly visualized non-invasively in vivo ${ }^{[5]}$. The blood vessels in the retina branch a number of times each time forming a vessel tree that is similar in characteristics to the branch it came from. Systems that have self-similarity at multiple scales are known as fractals. There are some reports of measuring fractal properties of blood vessel patterns of the retina; most have involved manual segmentation of the blood vessel patterns [6-8]. Now with reliable automated vessel segmentation, attention is turning to analyzing the retinal vasculature as a fractal $^{[9]}$

Fractal objects are self-similar structures that retain a similar level of complexity across all scales. For example, blood vessels repeatedly subdivide downstream into smaller blood vessels with similar network patterns. Fractal dimension (Df) quantifies the degree of complexity into a single value and is particularly useful for quantifying non-Euclidean geometric shapes such as vascular networks. The retinal circulation is a fractal object ${ }^{[10-12]}$, and fractal analysis has been used to study 
the embryological development of the retinal vasculature ${ }^{[11]}$ and vascular changes associated with diabetic retinopathy ${ }^{[12-}$

16]. Fractal analysis of vasculature formed in human retinal image can be used as a non-invasive technique for detection of early retinal vascular diseases. It is a more useful descriptor that offers a new language for examining the complex patterns found in ophthalmic practice.

\section{METHODS \& MATERIAL}

For our experiments we made use of the retinal images from DRIVE database ${ }^{[17]}$. From set of 40-40 images of healthy and diabetic subject images, we randomly selected 15 images of healthy subjects and 15 images of diabetic subjects. For our proposed system we designed a GUI in MATALB 2012 as shown in Fig. 1.

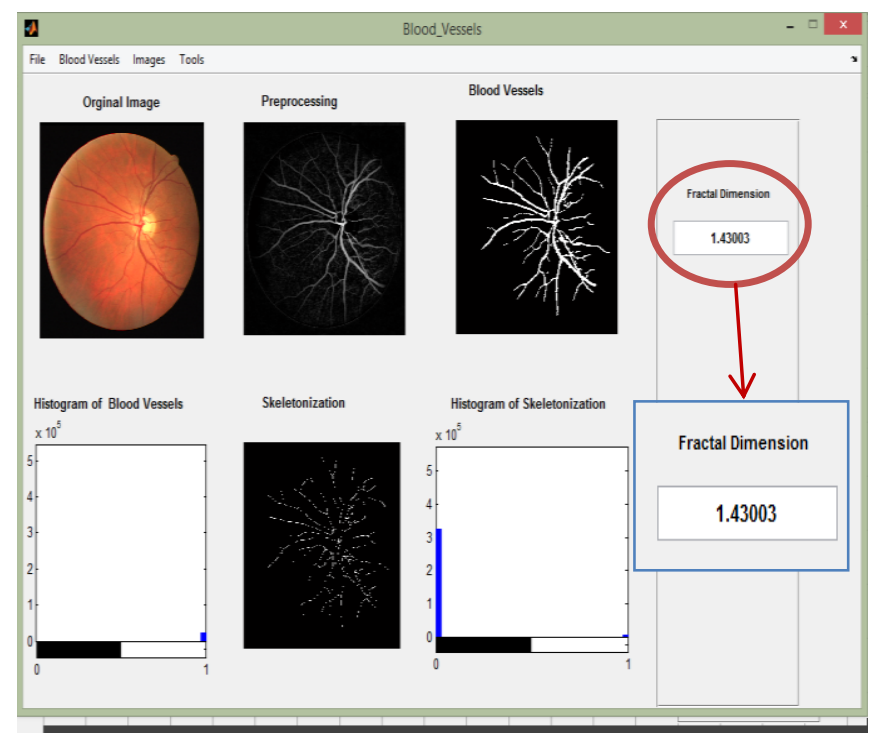

Fig-1: GUI showing the steps involved in fractal dimension calculation of a retina image

Fig 1 shows the sequentially performed steps to obtain the fractal dimension of retinal vasculature and analyze the complexity of blood vessels pattern. The high resolution colour fundus images obtained from DRIVE database were preprocessed by applying two filters. Firstly the green channel was extracted from the RGB image because green channel shows high intensity as compare to red and blue channels. Then in the next step 2D median filter was applied to remove noise from the images. And finally the preprocessed image was converted to grey scale. Further histogram equalization operation was performed to increase the contrast in the image. Once the image was enhanced threshold operation was performed to extract the blood vessels. These extracted blood vessels were used to calculate the fractal dimension.

For calculating fractal dimension, image segmentation was done using box counting technique. Box counting is one of the standard techniques employed in fractal studies. Given shape or pattern under study, is covered by boxes of different size (r) and the number of boxes required to cover the complete shape is recorded as $\mathrm{N}$. If the pattern has self-similarity and scale invariance, the plot of $\log (\mathrm{N})$ versus $\log (\mathrm{r})$ is a straight line indicating that the power law is obeyed this confirms fractal character. Fractal dimension is estimated from slope of this best fitting straight line and fractal dimension is equal to slope.

\section{RESULTS \& DISCUSSIONS}

Images of Retina of human subjects were studied to explore the degree of complexity associated with the patterns of blood vessel network in human eye to compare the texture for diabetic patients with those of healthy individuals selecting fifteen subjects for each. The shapes of patterns of blood vessel distribution are highly complex and very much differ from individual and thus it is very difficult to compare the structure with some standard in order to arrive at discernable difference. Fractal geometry is known to be effective in characterization of irregular shapes of this type thus the concept of fractals and fractal dimensions is employed for characterization of these patterns. In general higher the fractal dimension of an irregular shape, higher is the degree of complexity associate with the shape.

For the purpose of characterization of the irregular shapes from images of the Retina, high resolution images were used with identical resolution for both the healthy and diabetic subjects. Fractal dimension was obtained and used to characterize the patterns in terms of complexity of structure and the fractal dimension was estimated using box counting technique that best suits such applications.

Box counting technique makes use of square boxes of different sizes ( $r$ ) and the number of boxes $(\mathrm{N})$ of each size required to completely cover the image are determined. A graph is plotted taking $\log (\mathrm{r})$ at the axis of $\mathrm{x}$ and $\log (\mathrm{N})$ on the y-axis. If the image being analyzed is a fractal then there is scale invariance and self-similarity and the resulting plot is a straight line with a slope equal to the fractal dimension (with a change of sign) of that pattern or shape.

As the images used are colored it is convenient to process the image to improve the quality for the extraction of the pattern and convert it to black and white two color images in the form of bit map for further use. The image processing and counting of number of boxes of different size required to completely cover the image was implemented in Matlab and necessary software with GUI was developed for this purpose. Two typical images each for healthy subjects are shown in Fig. 2 along with the final images converted to two color bitmaps. 

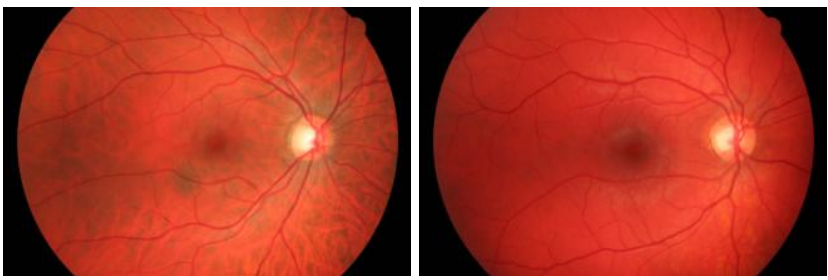

a
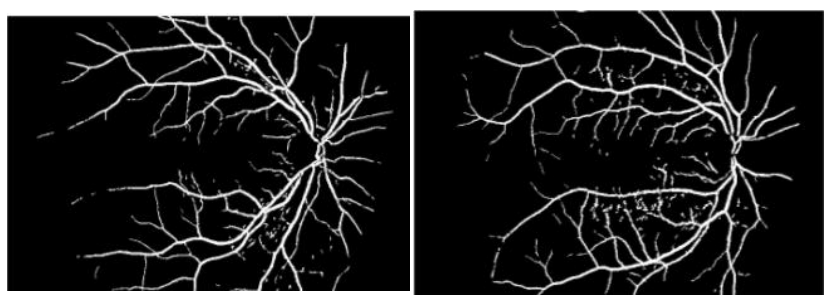

c

d

Fig -2: Typical images of Retina for healthy subjects $(a, b)$ and their two color bitmaps (c, d)

Two typical images of retina for diabetic subjects and the corresponding two color bitmaps are shown in Fig. 3. It can be seen from the comparison of Fig. 1 and 2 that there is more of structure and texture associated with the images for diabetic subjects adding to the complexity of shape. This complexity of shape and additional texture gives rise to a relatively higher fractal dimension and this can be used in distinguishing between the two.

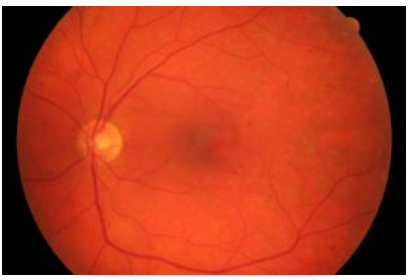

a

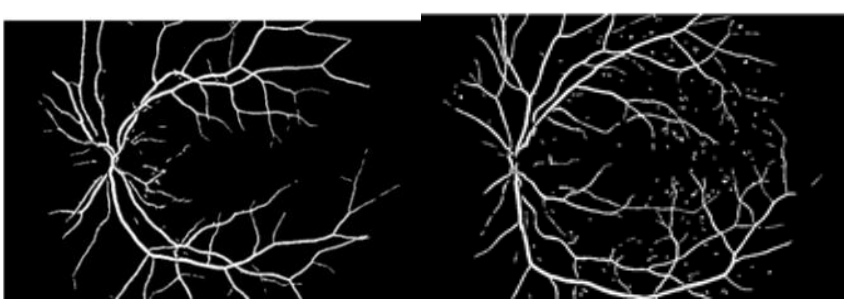

c

d

Fig -3: Typical images of Retina for diabetic subjects $(a, b)$ and their two color bitmaps (c, d)

Analysis of these images yield interesting results showing that the complexity of shape associated with the images for diabetic subjects is more than that of healthy subjects. The results of analysis of Box counting for two typical cases for healthy subjects are presented in Table -1 and 2 .

Table - 1: Results of Box counting of image for healthy subject (Case-A)

\begin{tabular}{|c|c|c|}
\hline S.No & $\log (\mathrm{r})$ & $\log (\mathrm{N})$ \\
\hline 1 & 0.0000 & 4.9193 \\
\hline 2 & 0.3010 & 4.3925 \\
\hline 3 & 0.6021 & 3.9113 \\
\hline 4 & 0.9031 & 3.4735 \\
\hline 5 & 1.2041 & 3.0781 \\
\hline 6 & 1.5051 & 2.6875 \\
\hline 7 & 1.8062 & 2.2765 \\
\hline 8 & 2.1072 & 1.8129 \\
\hline 9 & 2.4082 & 1.3010 \\
\hline 10 & 2.7093 & 0.7782 \\
\hline 11 & 3.0103 & 0.3010 \\
\hline 12 & 3.3113 & 0.0000 \\
\hline
\end{tabular}

Table - 2: Results of Box counting of image for healthy subject (Case B)

\begin{tabular}{|c|c|c|}
\hline S.No & $\log (\mathrm{r})$ & $\log (\mathrm{N})$ \\
\hline 1 & 0.0000 & 4.9334 \\
\hline 2 & 0.3010 & 4.4080 \\
\hline 3 & 0.6021 & 3.9296 \\
\hline 4 & 0.9031 & 3.4955 \\
\hline 5 & 1.2041 & 3.1004 \\
\hline 6 & 1.5051 & 2.7126 \\
\hline 7 & 1.8062 & 2.2945 \\
\hline 8 & 2.1072 & 1.8451 \\
\hline 9 & 2.4082 & 1.3424 \\
\hline 10 & 2.7093 & 0.7782 \\
\hline 11 & 3.0103 & 0.3010 \\
\hline 12 & 3.3113 & 0.0000 \\
\hline
\end{tabular}

The results from box counting shown in Table I and II are plotted as $\log (\mathrm{N})$ versus $\log (\mathrm{r})$ plot in Fig. 4 and 5 respectively. The points plotted as small circles are actual data and the bold ling joining the points is the best fitting line (least square fit) for the data points and the equation shown in the inset is the equation of this straight line. It is seen that all the points lie along a straight line which is also evident from the value of $\mathrm{R}^{2}$ that lies close to unity. This confirms the existence of self-similarity and scale invariance showing that the patterns do exhibit fractal character. 


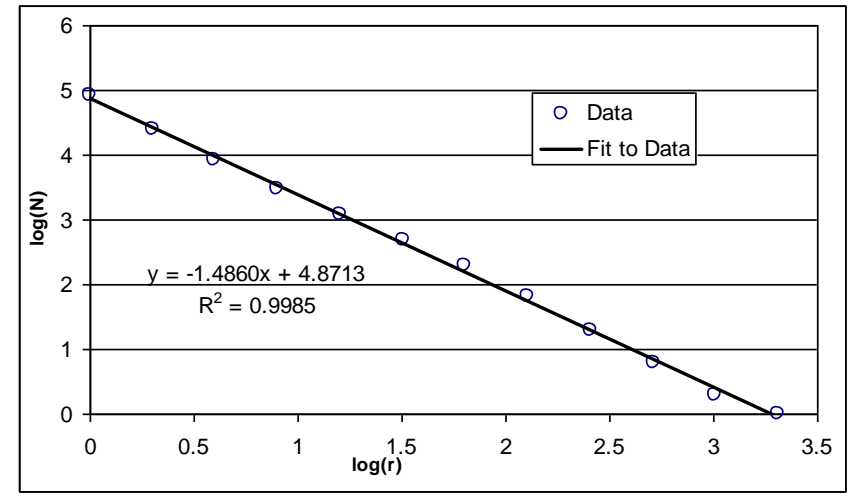

Fig-4: $\log (\mathrm{N})$ versus $\log (\mathrm{r})$ plot for Table I showing a fractal dimension of 1.486

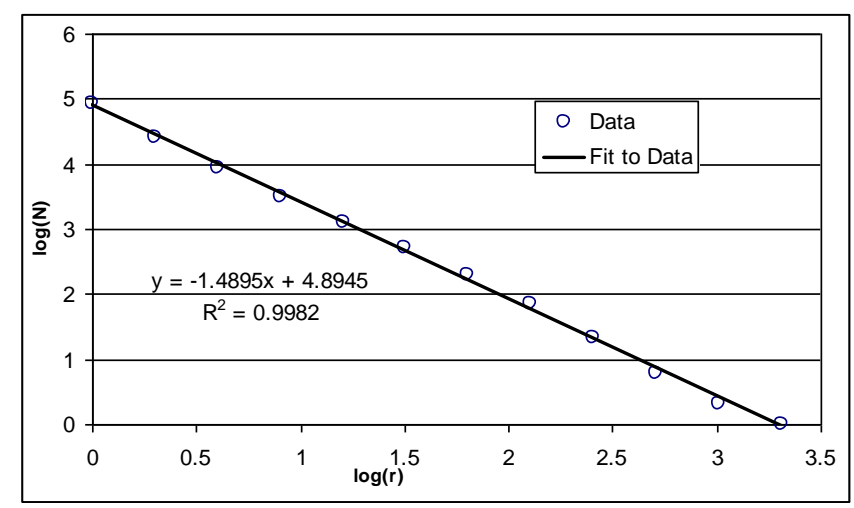

Fig-5: $\log (\mathrm{N})$ versus $\log (\mathrm{r})$ plot for Table II showing a fractal dimension of 1.4895

The results of analysis of Box counting for two typical cases for diabetic subjects are presented in Table 3 and 4.

Table - 3: Results of Box counting of image for diabetic subject (Case A)

\begin{tabular}{|c|c|c|}
\hline S.No & $\log (\mathrm{r})$ & $\log (\mathrm{N})$ \\
\hline 1 & 0.0000 & 5.0485 \\
\hline 2 & 0.3010 & 4.5373 \\
\hline 3 & 0.6021 & 4.0739 \\
\hline 4 & 0.9031 & 3.6545 \\
\hline 5 & 1.2041 & 3.2574 \\
\hline 6 & 1.5051 & 2.8549 \\
\hline 7 & 1.8062 & 2.3945 \\
\hline 8 & 2.1072 & 1.8692 \\
\hline 9 & 2.4082 & 1.3617 \\
\hline 10 & 2.7093 & 0.7782 \\
\hline 11 & 3.0103 & 0.3010 \\
\hline 12 & 3.3113 & 0.0000 \\
\hline
\end{tabular}

Table - 4: Results of Box counting of image for diabetic subject (Case B)

\begin{tabular}{|c|c|c|}
\hline S.No & $\log (\mathrm{r})$ & $\log (\mathrm{N})$ \\
\hline 1 & 0.0000 & 5.0840 \\
\hline 2 & 0.3010 & 4.5599 \\
\hline 3 & 0.6021 & 4.0818 \\
\hline 4 & 0.9031 & 3.6461 \\
\hline 5 & 1.2041 & 3.2355 \\
\hline 6 & 1.5051 & 2.8254 \\
\hline 7 & 1.8062 & 2.3483 \\
\hline 8 & 2.1072 & 1.8261 \\
\hline 9 & 2.4082 & 1.2788 \\
\hline 10 & 2.7093 & 0.7782 \\
\hline 11 & 3.0103 & 0.3010 \\
\hline 12 & 3.3113 & 0.0000 \\
\hline
\end{tabular}

The data from box counting from Table III and IV are plotted as $\log (\mathrm{N})$ versus $\log (\mathrm{r})$ plot in Fig. 6 and 7 respectively. The points plotted are actual data from box counting and the ling joining the points is the best fitting line for the data and the equation in the inset is the equation of this best fitting straight line. It is seen that all the points lie along a straight line which is also evident from the value of $\mathrm{R}^{2}$ that lies close to unity. This confirms the existence of self-similarity and scale invariance showing that the patterns do exhibit fractal character.

It is clearly seen from Fig. 4 to 7 that the patterns from retina images do possess self-similarity and scale invariance that is a characteristic of fractal shapes. The complexity of shape associated with these patterns is given by the fractal dimensions. It is also seen that the fractal dimensions of the patterns of retina images for diabetic subjects are on higher side as compared to those of healthy subjects. To demonstrate the fact that the complexity of shape associated with diabetic subjects retina images is more than those of healthy subjects the fractal dimensions obtained for fifteen healthy subjects are compared with those of diabetic subjects in Fig. 8.

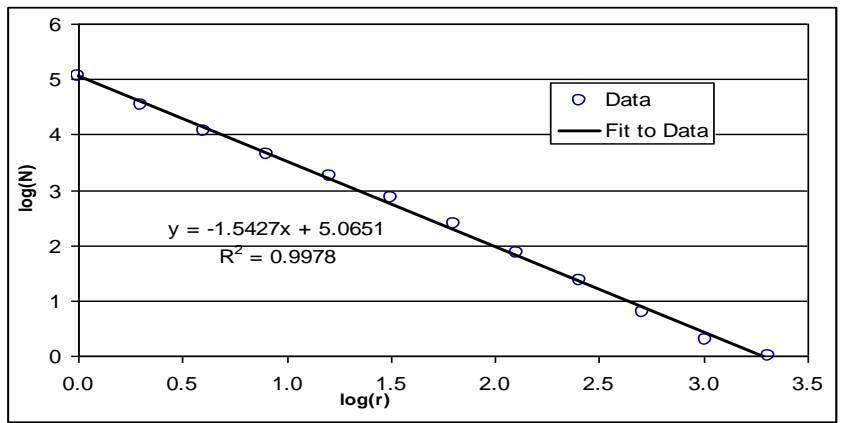

Fig-6: $\log (\mathrm{N})$ versus $\log (\mathrm{r})$ plot for Table III showing a fractal dimension of 1.5427 


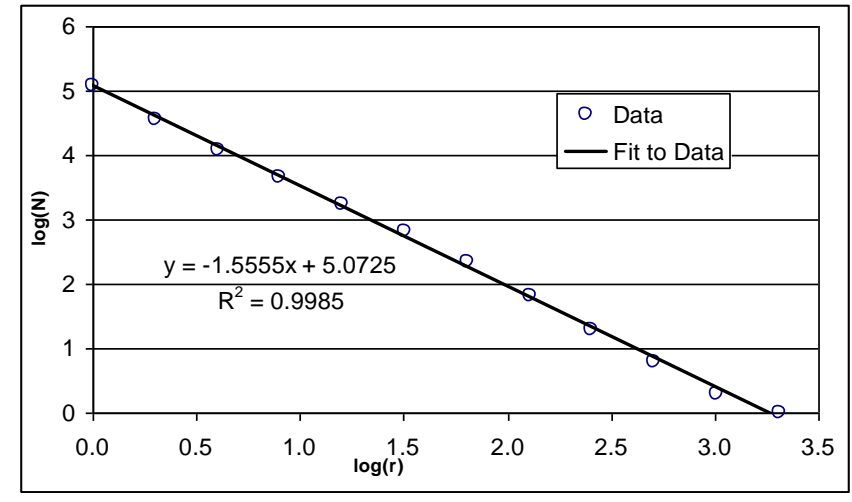

Fig-7: $\log (\mathrm{N})$ versus $\log (\mathrm{r})$ plot for Table IV showing a fractal dimension of 1.5555

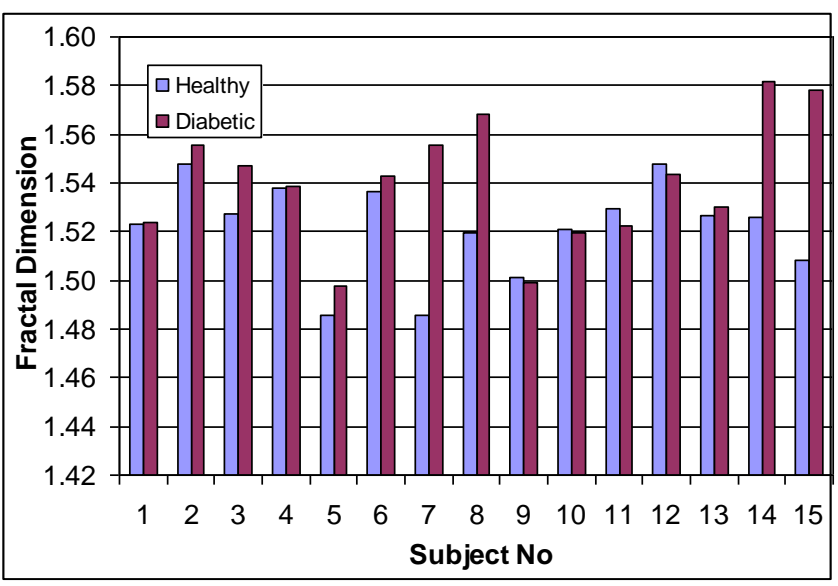

Fig-8: Comparison of fractal dimension of retina images of fifteen healthy and diabetic subjects.

Comparison of fractal dimension clearly reveals that the fractal dimension of retina images of diabetic subjects are higher compared to those of healthy subjects.

\section{CONCLUSIONS}

Diabetes causes gradual damage to retina resulting in serious deformation in arteries and veins in retina, this aspect is studied in terms of quantification of the degree of complexity associated with the distribution of blood vessels in eye. Retina images of fifteen healthy subjects are compared with those of fifteen diabetic subjects. It is fund that the increased complexity of structure and texture of the diabetic subjects results in a higher fractal dimension as compared to those of healthy subjects. Also the blood vessel patterns, both for healthy and diabetic subjects show self-similarity and scale invariance and hence the patterns are fractals. It is seen from Fig. 7 that fractal dimension can be used to distinguish diabetic subjects from healthy subjects and hence this technique could be used in diagnosis of diabetes using images of eye/retina. It is interesting that during other diagnostic procedures related to retina images, this information can be generated as additional information adding value to the diagnostic procedures.

\section{ACKNOWLEDGEMENTS}

The authors are thankful to UGC for providing financial support for the major research project entitled "Development of Color Image Segmentation and Filtering Techniques for Early Detection of Diabetic Retinopathy" F. No.: 41 $651 / 2012$ (SR). And are also thankful to DST for providing financial support for the major research project entitled "Development of multi resolution analysis techniques for early detection of non-proliferative diabetic retinopathy without using angiography" F.No. SERB/F/2294/2013-14. The authors also extend their gratefulness to Dr. Manoj Saswade, Director "Saswade Eye Clinic" Aurangabad and Dr. Neha Deshpande, Director "Guruprasad Netra Rungnalaya pvt. Ltd", Samarth Nagar, Aurangabad for providing the Database and accessing the Result.

\section{REFERENCES}

[1]. "Diabetic retinopathy". Mayo Clinic. http://www.mayoclinic.org/diseases-

conditions/diabeticretinopathy/basics/definition/con-20023311 [2]. Kertes PJ, Johnson TM, ed. (2007). Evidence Based Eye Care. Philadelphia, PA: Lippincott Williams \& Wilkins. ISBN 0-7817-6964-7

[3]. Tapp RJ, Shaw JE, Harper CA et al. (June 2003). "The prevalence of and factors associated with diabetic retinopathy in the Australian population". Diabetes Care 26 (6): 1731-7. doi:10.2337/diacare.26.6.1731. PMID 12766102.

[4]. Guidelines for the Comprehensive Management of Diabetic Retinopathy in India, A VISION 2020: The Right to Sight INDIA Publication, National Programme for Control of Blindness (NPCB), Directorate General of Health Services, Ministry of Health \& Family Welfare, Government of India. [5]. Niall Patton at. el., Retinal image analysis: Concepts, applications and potential, Progress in Retinal and Eye $\begin{array}{llll}\text { Research } & 25 & \text { (2006) 99-127, Elsevier, }\end{array}$ doi:10.1016/j.preteyeres.2005.07.001

[6]. Cheng, S.C., Huang, Y.M.: A novel approach to diagnose diabetes based on the fractal characteristics of retinal images. IEEE Trans. Inf. Technol. Biomed. 7(3), 163-170 (2003)

[7]. Cheung, N., Donaghue, K.C., Liew, G., Rogers, S.L., Wang, J.J., Lim, S.W., Jenkins, A.J., Hsu, W., Lee, M.L., Wong, T.Y.: Quantitative assessment of early diabetic retinopathy using

[8]. fractal analysis. Diabetes Care 32, 106-110 (2009), Masters, B.R.: Fractal analysis of the vascular tree in the human retina. Annu. Rev. Biomed. Eng. 6, 427-452 (2004)

[9]. G. Dougherty (ed.), Medical Image Processing: Techniques and Applications, Biological and Medical Physics, Biomedical Engineering, DOI 10.1007/978-1-4419-9779-1 11, (C) Springer Science+Business Media, LLC 2011 
[10]. Macgillivray TJ, Patton N, Doubal FN, Graham C, Wardlaw JM. Fractal analysis 2082 DIABETES CARE, VOLUME 32, NUMBER 11, NOVEMBER 2009 care.diabetesjournals.org of the retinal vascular network in fundus images. Conf Proc IEEE Eng Med Biol Soc 2007;2007:6456-6459

[11]. Mainster MA. The fractal properties of retinal vessels: embryological and clinical implications. Eye 1990;4:235-241

[12]. Masters BR. Fractal analysis of the vascular tree in the human retina. Annu Rev Biomed Eng 2004;6:427-452

[13]. Avakian A, Kalina RE, Sage EH, Rambhia AH, Elliott KE, Chuang EL, Clark JI, Hwang JN, Parsons-Wingerter P. Fractal analysis of region-based vascular change in the normal and non-proliferative diabetic retina. Curr Eye Res 2002;24:274-280

[14]. Daxer A. Characterisation of the neovascularization process in diabetic retinopathy by means of fractal geometry: diagnostic implications. Graefes Arch Clin Exp Ophthalmol 1993;231:681-686

[15]. Daxer A. The fractal geometry of proliferative diabetic retinopathy: implications for the diagnosis and the process of retinal vasculogenesis. Curr Eye Res 1993;12: 1103-1109

[16]. Daxer A. Mechanisms in retinal vasculogenesis: an analysis of the spatial branching site correlation. Curr Eye Res 1995; 14:251-254

[17]. J.J. Staal, M.D. Abramoff, M. Niemeijer, M.A. Viergever, B. van Ginneken, "Ridge based vessel segmentation in color images of the retina", IEEE Transactions on Medical Imaging, 2004, vol. 23, pp. 501-509.

\section{BIOGRAPHIES}

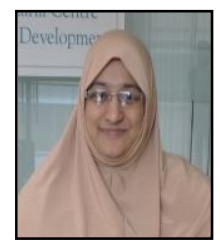

Nazneen Akhter, M.Phil. in Computer Science, is currently pursuing her doctoral programme in CS \& IT Dept. of Dr. B. A. M. University. She is at present having BSR Research Fellowship, previously she has served for 12 years as Asst. professor and even held the responsibility of Head of Dept., In her whole tenure she has served various reputed higher educational institutions and has also lend her services to one of the esteemed university in Saudi Arabia. She has 03 books with ISBN number to her credit. Her research interest includes biomedical signal processing, Embedded System design, Biomedical Engineering, Pattern Recognition and Biometrics. She has presented more than 16 papers in national and international conferences and she has more than 10 papers published in reputed journals.

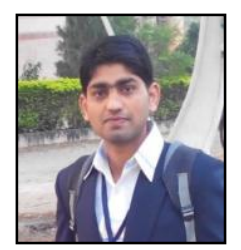

Yogesh M. Rajput has completed M.Sc. Computer Science in 2012 and presently working as a Project Fellow in Department of Computer Science and Information Technology, Dr. Babasaheb Ambedkar Marathwada University, Aurangabad. His area of specialization is Bio-Medical Image Processing and
Diabetic Retinopathy, He has published around 10 papers in reputed Journals and Conferences.

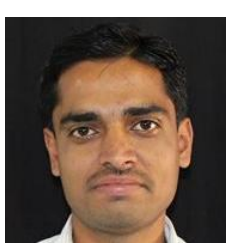

Sumegh shrikant Tharewal M.Sc. (computer science) BSR Research fellow at Dr. Babsaheb Ambedkar Marathwada University Aurangabad, Ex Assistant professor at Vivekanand College Aurangabad.He has published two research papers in state level and national level conference. His research area of interests is Digital image processing, Biometrics, cloud computing, Embedded system.

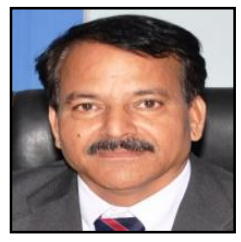

Dr. K. V. Kale, M.Sc., MCA Ph.D. FIETE, presently working as a Professor and head, CS \& IT Dept., Dr. B. A. M. University, Aurangabad, MS-India. He is a fellow of IETE, Life member of CSI, IAPR, ISCA, IEEE and elevated to senior member of IEEE. He is a member \& Faculty of Board of Studies of various universities in India \& has designed and developed new courses in computer science at UG \& PG level. He is recipient of VIJAY SHREE Award. He is actively engage in research and development and has around 235 research papers in reputed national/international journals and conferences and 05 books to his credit. He has produced $21 \mathrm{Ph}$. D. students and 04 M.Phil students under his able guidance. He is reviewer and editor of several journals of India and aboard. His research interest includes Image Processing, Pattern Recognition, Biometrics, Bioinformatics, S/W Egg., Artificial Intelligence, Computer vision, Neural Networks. He has completed three major Research projects \& currently working on multimodal Biometrics \& Remote Sensing.

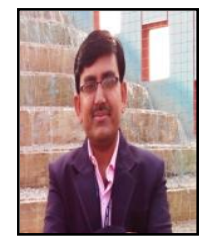

Dr. Ramesh R. Manza is NET, SET qualified and is Ph.D in Computer Science and is currently working in the capacity of Asst. Prof. in CS \& IT Dept. of Dr. B. A. M. University, Aurangabad, India. His area of specialization includes Bio-Medical Image Processing, Computer Vision, Nano-robotics, MEMS and Biometrics. He has presented around 105 papers at national and international conferences and around 42 papers in reputed Journals. He is leading Two Major research projects sanctioned by UGC and DST on Diabetic retinopathy. Total 16 students have been awarded M. Phil. under his guidance and 8 students are leading their Doctoral program. He is author of 05 Books and is on editorial board of 03 International Journals. He has been coordinator, treasurer and organizing secretary of 05 International Conferences and 19 workshops and trainings. 